\title{
Memory and preference for the colors of objects
}

\author{
PATRICIA SIPLE \\ Wayne State University, Detroit, Michigan \\ and \\ ROBERT M. SPRINGER \\ AT\&T Information Systems, Holmdel, New Jersey
}

\begin{abstract}
Memory for object color often produces object-specific deviations from actual color. Several studies have indicated that "memory colors" exist and in some cases influence perception of object color. Systematic changes in memory for color per se cannot account for these memorycolor phenomena. A study was conducted to characterize more specifically the nature of memory for object color information. The study was designed to assess the dependence of memory color on shape and texture information, to compare memory color with color preference, and to determine whether sophistication about color technology affects color memory and preference. Results indicated that, for hue and brightness, memory and preference were quite accurate for the objects tested; however, all subjects remembered and also preferred all items to be more highly saturated. Change in context produced no change in accuracy, suggesting that access to memory for object color is independent of shape and texture information. Color seems to be an independently accessible feature of memory representation rather than an integral part of a prototypic representation.
\end{abstract}

The concept of "memory colors" was introduced by Hering to explain color constancy in perception; yet, a century later, the nature of memory colors is not well understood. According to Hering (1964), the most typical color of an object becomes a component of the memory representation for that class of objects and influences our perception of object color unless we particularly attend to the color itself. Katz (1935) has added that we also often exaggerate salient aspects of color, especially when those aspects have been given labels (e.g., picking out a red that is too saturated as the color of a brick).

Several studies showing an influence of memory color on perception have validated Hering's claim that we retain and employ representative colors for often-seen objects. When an object of a known color is illuminated such that its actual color of presentation is not the color identified with it, observers' color matches are influenced by the memory color of the object (Adams, 1923; Bruner, Postman, \& Rodrigues, 1951; Duncker, 1939; Harper, 1953). Comparison of these matching differences with the

This study was carried out while the first author was under contract with Xerox Corporation, Research Division, Rochester, New York, and a member of the Psychology Department at the University of Rochester. Robert Springer was then an employee of Xerox Corporation. The authors are grateful to David Feldman for the preparation of stimuli, to Steven Silverstein for carrying out the study and the data analysis, and to Warren (Dusty) Rhodes for his guidance, support, and assistance throughout the project. Requests for reprints should be sent to Patricia Siple, Department of Psychology, Wayne State University, Detroit, Michigan 48202. results of studies of matches to patches of color indicates that object-color-match differences cannot be accounted for by sensory and methodological factors (Newhall, Burnham, \& Clark, 1957; Springer, 1969; Tate \& Springer, 1971). Clearly, our representations of objects in memory contain information about color that can affect our perceptions of objects.

A number of studies have attempted to directly assess memory colors and compare them with actual object colors. In a supplemental report to their primary investigations, Newhall et al. (1957) report a study conducted by Newhall and Pugh in which memory colors for familiar objects and substances were determined. Subjects were asked to choose from the set of Munsell colors the one that best represented the colors of the object named. When these selections were compared with actual colors for the items tested, hue, saturation, and brightness differences were found. Memory hues moved toward the dominant hues associated with the items (e.g., green grass, yellow sand); there was a tendency for memory colors to be more saturated, but some items (e.g., sand, skin, and concrete) did not show this effect; and, with the exception of the memory color for brick, brightnesses averaged higher by slightly more than a Munsell step. Similar results are reported by Bartleson (1960).

Other studies have attempted to determine color preferences for objects by asking subjects to rate a set of differently illuminated objects (Sanders, 1959) or to rate or choose the preferred of a set of photographs (Bartleson, 1959; MacAdam, 1951). In these studies, 
differences between preferred and actual colors have been noted. However, these authors and others (e.g., Judd, 1967; Thornton, 1974) have seemed to confuse, or equate, memory with preference, often using both words interchangeably. Memory color and preference judgments have been compared by Bartleson (1959; Bartleson \& Bray, 1962). Those studies indicate that memory and preference colors should not be assumed to be equivalent, and that there may not be a simple relationship between the two. While the preferred and memory-color hues for natural Caucasian flesh were found to be the same (except more yellow than actual flesh), preferred hues for blue sky and green grass were found to be closer to the actual hues than to memory-color hues.

As with earlier results, these differences between actual and memory colors cannot be explained by sensory factors related to color perception. Bartleson (1961) has directly compared actual object colors, memory colors for those objects, and successive color matches to unlabeled patches of those colors. He concluded that while successive color matching produced lawful changes in saturation and brightness, memory colors showed unsystematic shifts in all three color dimensions, hue, saturation, and brightness. Other data also suggest that there is considerable consistency of memory color for a particular object, relative to its actual color; however, the nature of the color shift seems specific to the object. For example, viewers prefer more saturation for Caucasian flesh, less saturation for Japanese flesh, and hue changes for both (Sugimoto, Ezawa, \& Tamura, 1973). Even for color patches, there is a tendency for memory over a long duration to move toward "impressive" characteristics (e.g., an increase in brightness for brighter colors, but a decrease for darker colors; Hanes \& Rhodes, 1959).

Because of task and methodological differences among studies, and the small number but wide range of objects examined, few conclusions can be drawn concerning the nature of memory colors. The following study was designed to systematically investigate remembered and preferred colors for a specific set of food objects: fruits and vegetables. It is possible that systematic color-memory shifts may exist for classes of objects, but differ from class to class. Studies have differed in the nature of cues given to aid in color choice. In some cases, only the name of the object was given and the task was to choose from among a set of color patches, whereas in other studies involving the selection of color pictures or objects, both shape and texture information was combined with the color information. These different conditions should produce different results if form and color are integrally related (Garner, 1974). In the present study, the provision of form and of both form and texture cues was compared with a condition in which only the name of the object was provided. If color and form are interdependent in memory, then supplying shape and texture information should facilitate the choice of color either in the specific color chosen or in the variability range for the chosen color. Alternatively, shape and texture cues should have little effect on memory color if color is accessible as an independent attribute.

Both remembered and preferred colors were determined for each of our objects. As noted above, memory and preference have often been assumed to be equivalent. Yet, depending on the nature of the representation, color information residing in memory may be operated on differently by response and decision processes to produce different colors for memory and preference tasks. Bartleson and Bray (1962) have suggested that context may be important in the determination of memory and preference colors. Memory colors have been chosen from color patches, whereas preferred colors have been based on choices among photographs of objects or the objects themselves. A comparison of memory and preferred colors with and without shape and texture cues for the object should help to clarify the role of context in color choice.

Finally, several investigators have indicated that there are individual differences in susceptibility to color-memory phenomena (e.g., Duncker, 1939; Springer, 1969). In Duncker's study, 3 of 11 subjects produced accurate matches of off-color objects, and these subjects were more sophisticated about color properties (one was a painter, for example). Also, in Bartleson's studies comparing memory and preferences, all preference data were obtained from persons with technical backgrounds in color reproduction technology. In the present study, we have compared experts in color technology with subjects naive in this field.

\section{METHOD}

\section{Subjects}

Three groups of six adults with normal or corrected vision served as subjects. All were tested and found to have no deficiencies in color vision. One (expert) group consisted of six Xerox Corporation employees skilled in color reproduction technology. Two other (naive) groups with little or no knowledge of color technology were hired through an agency and paid for their participation in the study. These two naive groups were used to compare two different procedures.

\section{Materials and Apparatus}

Color selections were made with regular binocular vision under daylight viewing conditions. Color adjustments were produced with a Burnham-type colorimeter (Burnham, 1952), fitted with a mechanism allowing placement of a $19 \times 22 \mathrm{~cm}$ surround over the viewing area. The colorimeter was calibrated daily so that data could be transformed to CIE coordinates and Munsell values for hue, value, and chroma. ${ }^{1}$

Stimuli for the study were made from sets of photographs of six individual fruits and vegetables (carrot, corn, lettuce, lime, orange, and peanut) photographed to delete highlights and reproduce the 
colors as accurately as possible. The six fruits and vegetables appeared individually in the center of the photograph on a fairly neutral background (Munsell 9.17 PB/5.60/.29) surrounded by other fruits and vegetables not used as test items. A sample photograph reproduced in black and white is shown in Figure 1. Other photographs were constructed with no item in the center area.

As a test of the accuracy of our color reproductions, Munsell matches to the photographs were compared with Munsell matches to actual fruits and vegetables in two supermarkets containing a large array of produce. For each of the fruits and vegetables, care was taken to record the average match to a large sample containing variations in color. All matches were made under an approximately $5,000^{\circ} \mathrm{K}$ lamp by superimposing the color chip over either the fruit or vegetable or a photographic reproduction. The differences between the matches to actual fruits and vegetables and the photographs were computed. The average differences in Munsell units with $95 \%$ confidence intervals were $-0.1 \pm 2.2,0.4 \pm 0.6$, and $0.4 \pm 0.4$ for hue, value, and chroma, respectively. Thus, the photographic stimuli accurately reproduced the colors of the fruits and vegetables.

Stimuli were constructed from these photographs and from transparencies of the photographs to allow for three separate viewing conditions: disk, silhouette, and texture. Silhouette stimuli were constructed by carefully cutting out the central objects from one set of six pictures. These could be placed over the colorimeter such that a hole in the shape of the fruit or vegetable appeared over the viewing area. To produce the disk condition, the area of each fruit and vegetable was computed and circles of equal area were cut in the centers of six photographs with no central object. Finally, for the texture condition, a transparency of each original photograph was placed behind the appropropriate silhouette photograph so that the texture of the object could be seen through the hole, and these two together were placed over the viewing area of the colorimeter. To equate the disk and silhouette conditions with the texture condition, the density of each transparency of the six items was determined and neutral-density filters approximating the density of the objects in the transparencies were placed over the colorimeter viewing area when these conditions were tested.

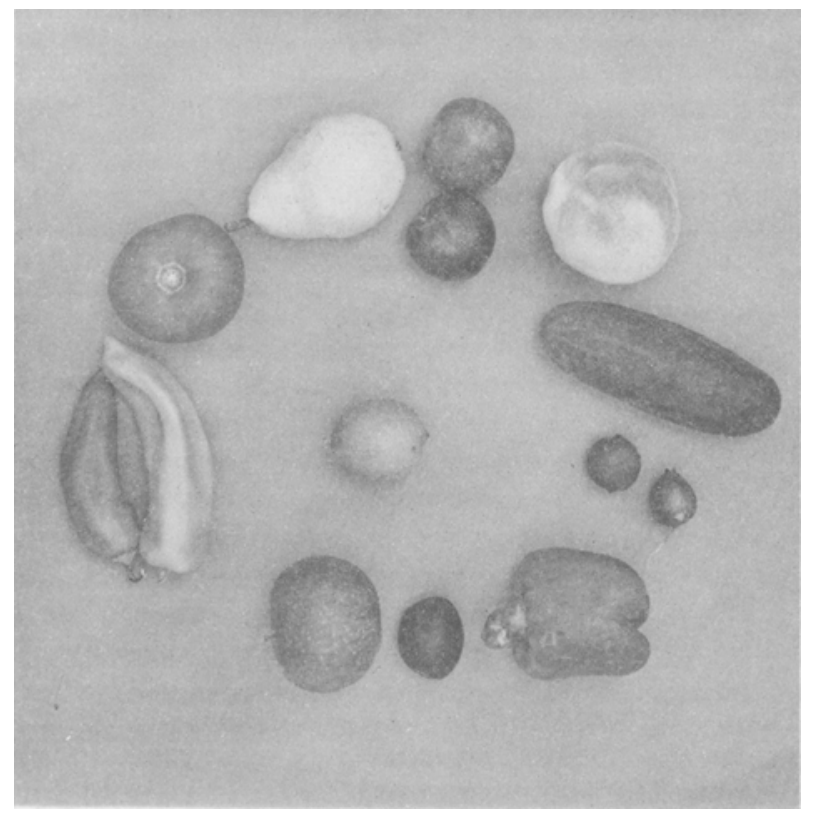

Figure 1. A black-and-white reproduction of the photograph from which materials for the fruit lime were constructed.

\section{Design and Procedure}

Each session began with the subject's producing his/her best matches to a white card, which served as a reference white for the determination of CIE coordinates for all further color selections. The subjects were allowed to adjust the three knobs of the colorimeter until they were satisfied with the selected color. However, to avoid problems of adaptation and afterimages (as outlined by Springer, 1969), the subjects were allowed to work at the task of color selection for intervals of $15 \mathrm{sec}$, followed by 5 -sec rest intervals when they were instructed to look at a large sheet of neutral gray paper. Time intervals were signaled by a buzzer which cycled through 15- and 5-sec intervals. The subjects were told to change what they were doing each time they heard the buzzer. When a satisfactory match was achieved, the colorimeter was adjusted randomly to a different color. Two additional matches were achieved in the same way. This procedure was used to determine the subject's best match to reference white and for all other color selections.

Following the match to white, the subjects were asked to select colors for the series of fruits and vegetables as they remembered the fruits and vegetables to be, typically, on the average (memory instructions). The three viewing conditions (disk, silhouette, and texture) provided three levels of contextual cuing to aid the subject. Practice for each of these context conditions was given with the fruit strawberry. Then, for one naive group and the expert group, the six experimental fruits and vegetables were presented in a different order to each subject. For each item, the three contextual conditions were presented one after the other with the order counterbalanced for each group. Because we were concerned that presentation of the three contextual conditions together might weaken the effect of that variable, a second naive group received the 18 test stimuli arranged such that only one contextual condition for each item appeared in each third of the test stimuli. Again, order of presentation of contextual conditions was counterbalanced, and the fruits and vegetables were ordered randomly. Three satisfactory matches were produced for each test stimulus. A break of 10-15 min followed.

In a second session, subjects were told that the colors they remembered might not be what they would like the fruits and vegetables to look like. They were asked this time to select the colors they would like them to be, or prefer them to be (preference instructions). Subjects first matched the white card and were given practice with the strawberry conditions. Then the 18 test stimuli were presented, as they had been for the memory session.

In a final session, the subjects were shown the pictures from which the stimuli were constructed. For each test item, the original picture was placed in a holder above the photograph over the colorimeter and the subjects were instructed to match the item pictured as accurately as possible. The procedure followed was identical to that for the memory and preference sessions. These matches were taken as a baseline from which preference and memory deviations could be assessed. Including breaks between sessions, the subjects needed 3 to $4 \mathrm{~h}$ to complete the three sessions.

\section{RESULTS AND DISCUSSION}

Colorimeter data were converted to Munsell notation for analyses. Average hue, value, and chroma were computed for each subject for each experimental condition. For each subject, each matching, or control, measurement was subtracted from the appropriate memory and preference measurements for each of the three color components (hue, value, and chroma). This yielded difference scores for each condition for both memory and preference. These differ- 
ence scores served as the basic data for analysis. Separate analyses of variance were performed for the three color components: hue, value, and chroma. Each of these repeated-measures analyses compared groups (two naive and one expert), tasks (memory and preference), contextual cues (disk, silhouette, and texture), and items (six fruits and vegetables).

In addition to analyses of means, analyses of intrasubject variability were carried out. ${ }^{2}$ Separate variances for hue, value, and chroma were computed over the three repetitions for each subject for each condition. These measures were submitted to identical analyses as were the means, with the exception that difference scores were not formed and the matching condition was compared directly with the memory and preference conditions. For all analyses, a .01 level of significance was maintained.

\section{Hue}

Means analysis. Mean hue differences combined over groups are shown in Figure 2. Hue differences are reported in Munsell units and $95 \%$ confidence intervals are shown for each point. It can be seen immediately that memory for hue was remarkably accurate when compared with the matching condition and that preferred hue did not deviate from the remembered hue. Only for the fruit lime is there even a tendency for deviation from the matching condition, and this tendency is not reliable. Such a result could easily be due to our choice of lime or to the subjects'

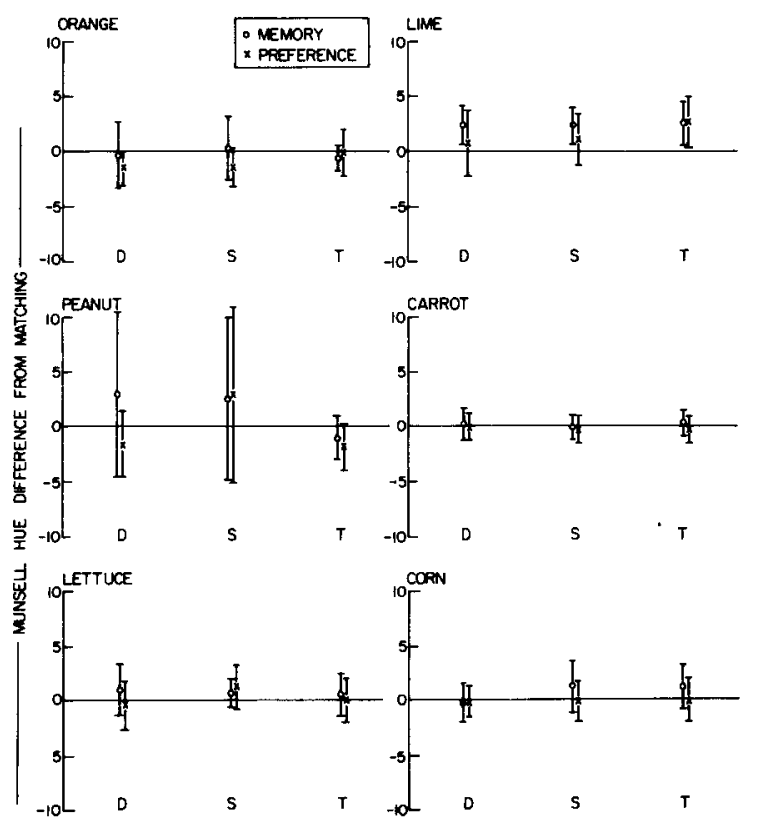

Figure 2. Mean hue differences from matching for memory and preference conditions as function of context $(D=d i s k, S=$ silhouette, $\mathbf{T}=$ texture). Mean differences, comblned over groups, are reported in Munsell units with $95 \%$ confidence intervals indicated for each mean difference score. lack of familiarity with limes, a comment often volunteered. Confidence intervals for peanut are in general larger than those for other fruits and vegetables, probably because it is highly desaturated, making a wider range of hues acceptable. As might be expected after examination of Figure 2, no differences were found among groups, and no other tests, including interactions, reached significance.

As noted earlier, the relationship between memory hue and actual hue seems to be unsystematic and specific to the items tested. Looking again at other studies, memory hues for foods seem to be quite accurate relative to other items that have been tested. Sanders (1959) found no hue differences for butter, tea, or potato chips; Bartleson (1961) found no differences for lemons or oranges; and we have found no differences for the six fruits and vegetables examined here. Although Sanders may have confused memory with preference, we have, in fact, shown that the memory hue is the preferred hue for these items. Both memory and preferred hues seem to be retrievable, independent of the kinds of contextual cues varied here: shape and texture. Finally, no differences were found between naive and expert groups.

Variance analysis. An analysis of the intrasubject hue variances derived from the three replications for each condition for each subject produced two significant results. Some fruits and vegetables produced higher variances than did others $[F(5,75)=5.77, p<$ .0011 , and these item differences varied as a function of the task being performed (memory, preference, or matching) $[F(10,150)=3.18, p<.001]$. Examination of the variances for this task $\times$ item interaction revealed that two unusually high mean variances (memory hue for lettuce and preferred hue for peanut) are the probable cause of this interaction and contribute to the main effect as well, yielding hue differences for those two vegetables that are twice those of the other four. Again, both lettuce and peanut are highly desaturated, making a greater range of hues acceptable. While there was a tendency for the naive group with mixed presentation orders to show a higher overall variance (4.86) than either the other naive group (2.74) or the expert group (1.45), group differences were not significant $[F(2,15)=2.88$, $\mathrm{p}<.09]$.

\section{Value}

Means analysis. Mean Munsell value differences with $95 \%$ confidence intervals are shown in Figure 3 combined over groups. The overall mean value difference did not vary from zero. An analysis of variance produced only one significant result, that for differences among fruits and vegetables $[F(5,75)=$ $3.29, p<.01]$. This item difference is primarily accounted for by data for peanut, which, as can be seen from Figure 3, is the only item whose preference and 


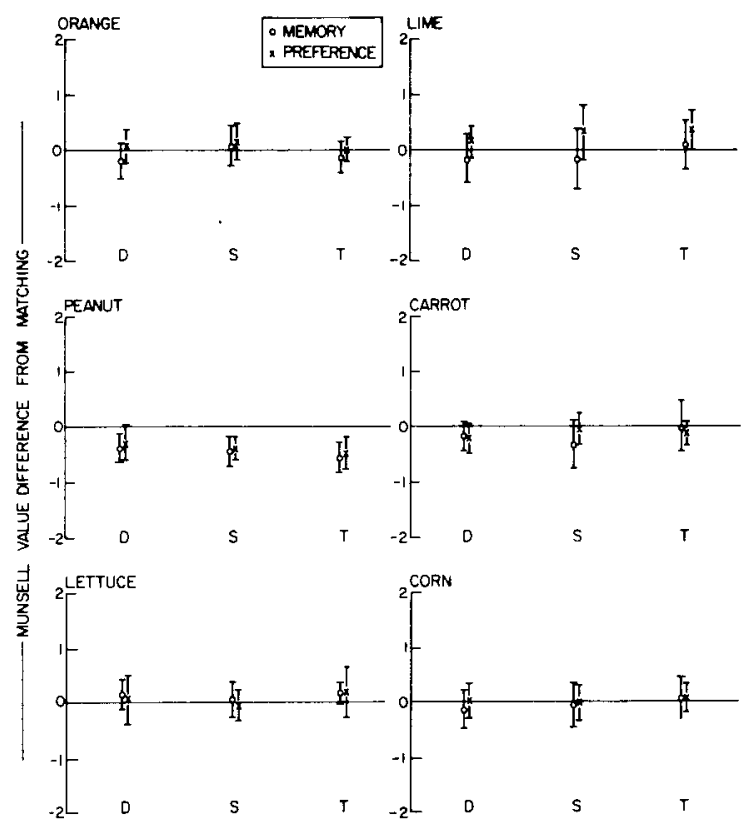

Figure 3. Mean value differences from matching for memory and preference conditions as a function of context $(D=$ disk, $S=$ sllhouette, $\mathrm{T}=$ texture). Mean differences, combined over groups, are reported in Munsell units with $95 \%$ confidence intervals indicated for each mean difference score.

memory value selections tended to deviate from matching. This deviation for peanut ( -.42 Munsell steps for memory value and -.35 for preference) is three times larger than the average deviation for any other fruit or vegetable.

Other studies have found differences between memory and actual color value. Newhall et al. (1957) found memory values to be an average of 1.1 Munsell steps greater than the brightness of natural objects, and Bartleson (1961), for a somewhat different set of objects, reports 7 of 11 values of natural objects to be greater than those of the memory colors chosen. When specific objects used in these studies are considered, however, memory color for value, like that for hue, is quite accurate for food items but for other objects tends to show differences specific to the object considered. Memory-value differences, when they exist for objects, do not show the systematic variation found for memory for color patches. Data from Hanes and Rhodes (1959), as well as those of Newhall et al. and Bartleson, indicate that for color patches memory for value increases for light colors and decreases for dark colors. Such consistency is not the case for object-color memory or preference.

Variance analysis. An analysis of variance of value variances yielded no significant main effects or interactions. Variances of value selections also were unrelated to the values measured for the photographed items.

\section{Chroma}

Means analysis. Mean Munsell chroma differences combined over groups are shown in Figure 4. It can be seen that both memory and preferred chroma were higher than matched chroma. An analysis of variance indicated that this effect was significant $[F(1,15)=$ 47.82, $\mathrm{p}<.001$ ]. Average preferred chroma $(2.33$ steps greater than matching) was half a Munsell step higher than memory chroma (1.84 steps greater than matching), but this difference did not reach significance $[F(1,15)=2.86, p<.12]$. Average chroma difference scores for individual fruits and vegetables differed significantly from each other $[F(5,75)=$ $9.96, \mathrm{p}<.001]$, varying from .87 steps for peanut to 4.06 for corn. Items with midrange chroma values (carrot, corn, and lime) showed greater differences than did those high (orange) or low (lettuce and peanut) in saturation. Other main effects and all interactions did not reach significance. Interestingly, experts showed just as large an average difference score (2.11 steps) as the comparably run naive group (2.03 steps) or the naive group with cue and item combinations intermixed ( 2.12 steps).

These data are in agreement with Bartleson (1961) and Newhall et al. (1957), who found memory chroma for objects to be greater than matched chroma (.95 steps) and measured natural chroma (2.72 steps), respectively. However, this enhancement of saturation in memory is not found for successive matches to color patches; Springer (1969) has shown that memory for the saturation of a color

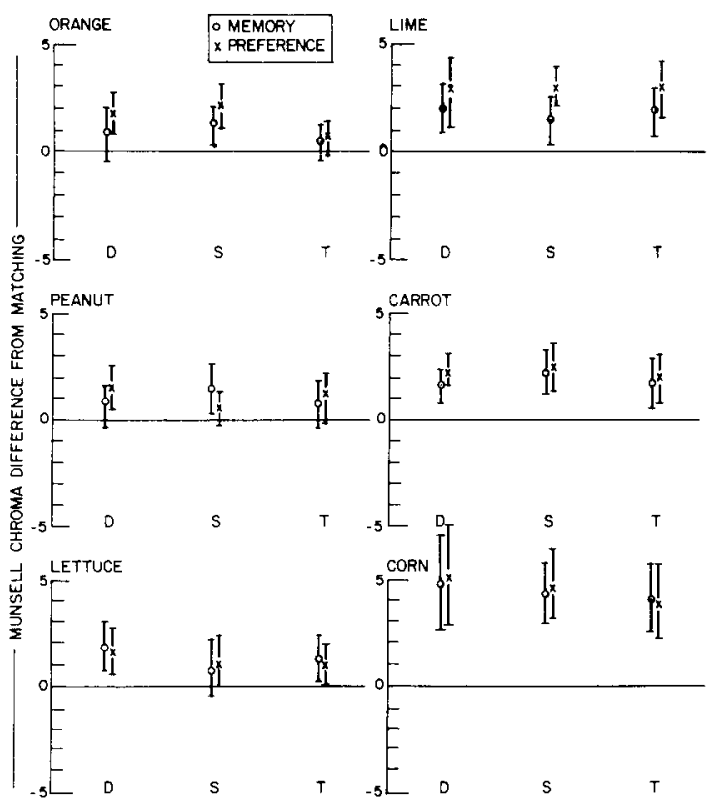

Figure 4. Mean chroma differences from matching for memory and preference conditions as a function of context $(D=$ diak, $S=$ sllhouette, $T=$ texture). Mean differences, combined over groups, are reported in Munsell units with $95 \%$ confidence intervals indicated for each mean difference score. 
patch showed no enhancement when at least $5 \mathrm{sec}$ had elapsed after presentation of the color standard. Studies reporting enhancement of saturation in memory for color patches (e.g., Newhall et al.) were shown by Springer to suffer from procedural and response bias factors. Memory and preference for increased saturation occurs only for objects, and not for color patches.

Variance analysis. An analysis of variance of chroma variances yielded only one significant result, an item $\times$ group interaction $[F(10,75)=3.78, p<$ $.001]$. The naive group with mixed presentation showed greater variability for some items, particularly corn and lettuce; and the expert group showed less variability for some items, especially carrot, orange, and peanut. Overall group differences did not reach significance $[F(2,15)=5.23, p<.02]$; however, the group means reflect the differences reported for the interaction: $\mathbf{2 . 1 2}$ for the mixed-presentation naive group and .83 and 1.37 for the expert and other naive groups, respectively.

\section{Accuracy of Memory Colors}

In the above analyses, difference scores were used to assess the accuracy of memory colors in order to reduce the effect of other possible systematic sources of variability. These sources include perceptual judgment effects, possible slight deviations produced by the CIE to Munsell transformations, and the effects of filters used in the disk and silouhette conditions to compensate for the texture gradients in the texture condition. Conclusions about the accuracy of memory colors rest on the accuracy of our photographic reproductions, and we have shown that they were quite accurate. Given the surprising accuracy of both memory and preference colors, however, a comparison of average memory and preference Munsell values with matched Munsell colors of selected representative actual fruit and vegetables is presented in Table 1.

Table 1 confirms the results of the means analyses presented above. Only chroma measures for memory and preference show large, systematic deviations from Munsell matches to actual fruits and vegetables. Hue differences from actual, ranging from -3.7 to 2.4 , are within the expected range, given the possibilities for error. Only a few representative items were chosen for matching, and the matching chips were often selected from sets whose members varied by several Munsell steps. In addition, hue judgments were the most variable in our data; $95 \%$ confidence intervals averaged \pm 2.34 Munsell steps for the difference scores shown in Figure 1. There is a tendency for memory- and preference-value measures to be lower than those of the actual items, a tendency opposite to that reported by Bartleson (1961) and Newhall et al. (1957). Value differences from actual values range from -1.5 to +0.4 units, with an average of 0.7 units. These differences are not reliable, however, when considered in the context of our pictures, which averaged $0.4 \pm 0.6$ Munsell units lower than actual values and difference scores, which had average confidence intervals of \pm 0.33 units. All memory and preference chroma measures were greater than those of actual items, ranging from +1.1 to +7.4 Munsell units, with an average difference of +3.2 units. These differences from actual measures are quite reliable when our pictures, which averaged $0.4 \pm 0.4$ units lower than actual values, and difference scores, with average confidence intervals of \pm 1.25 units, are taken into account.

\section{GENERAL DISCUSSION}

For a homogeneous set of food objects, the relationship between memory color and actual color matches is systematic. Object-color memory for hue and brightness is quite accurate; but, for chroma, all items are both remembered and preferred to be more highly saturated than they naturally are. When the food objects in other studies are considered separately, results support the relationship found here. Hue shifts reported by other investigators for such items as foliage, green grass, blue sky, flesh, and red brick are not reported for food items. Only saturation shifts are consistent across studies for this class of objects.

It is not clear why some classes of items show differences between actual and memory colors. It is possible that color may be an important enough attribute of foods for specific color information to be represented with the concept, whereas that for such items as brick or sky our ideals of the more salient color characteristics may suffice, leading to no specific storage of color information. Alternatively, the ef-

Table 1

Comparison of Memory and Preference Colors With Actual Colors

\begin{tabular}{|c|c|c|c|c|c|c|c|c|c|}
\hline \multirow[b]{2}{*}{ Item } & \multicolumn{3}{|c|}{ Hue } & \multicolumn{3}{|c|}{ Value } & \multicolumn{3}{|c|}{ Chroma } \\
\hline & Memory & Preference & Actual & Memory & Preference & Actual & Memory & Preference & Actual \\
\hline Carrot & $5.59 \mathrm{YR}$ & 5.29 YR & $3.13 \mathrm{YR}$ & 5.20 & 5.14 & 5.50 & 12.43 & 12.90 & 11.00 \\
\hline Corn & $2.60 \mathrm{Y}$ & $1.32 \mathrm{Y}$ & $5.00 \mathrm{Y}$ & 8.07 & 8.13 & 8.50 & 13.09 & 13.42 & 6.00 \\
\hline Lettuce & $5.87 \mathrm{GY}$ & $5.54 \mathrm{GY}$ & $5.00 \mathrm{GY}$ & 6.22 & 6.16 & 7.50 & 7.95 & 8.05 & 4.00 \\
\hline Lime & $6.33 \mathrm{GY}$ & $5.36 \mathrm{GY}$ & $5.00 \mathrm{GY}$ & 4.52 & 4.86 & 4.50 & 8.81 & 10.14 & 7.00 \\
\hline Orange & $7.50 \mathrm{YR}$ & 6.44 YR & $6.88 Y \mathrm{R}$ & 5.41 & 5.58 & 6.50 & 12.60 & 13.23 & 11.50 \\
\hline Peanut & $3.26 \mathrm{Y}$ & $1.55 \mathrm{Y}$ & $0.83 \mathrm{Y}$ & 5.16 & 5.24 & 6.67 & 6.20 & 6.25 & 4.00 \\
\hline
\end{tabular}


fect of color labeling, either explicitly or implicitly, when a task is performed may well affect the color chosen. That labeling can have a powerful effect is demonstrated in a study by Loftus (1977). Subjects who saw an accident with a green car present tended to most often choose the correct car color. However, subjects who were asked a question about the "blue car" immediately or 1 week later shifted their memories for the car's color toward bluer hues. Thus, memory at any moment in time reflects an integration of the subject's knowledge about the object, updated by subsequent information. In the studies of Bartleson $(1960,1961)$ and Newhall et al. (1957), many of the items tested were given color labels to help distinguish them (e.g., blue sky, green grass). Some food items tested may have hues closer to those hues chosen for their color labels (e.g., orange, lemon). While labeling may not account for all the results presented, it must be seriously considered in colormemory studies. We know that labeling a color patch moves the memory of the hue of that patch toward the hue label (Bornstein, 1976; Hendrick, Wallace, \& Tappenbeck, 1968).

Providing integrated shape and texture cues during color selection did not produce greater accuracy or lessen variability of color selection. This result suggests that access to memory for color is not dependent on the provision of integrated form and texture information, but that it is an independently accessible attribute. This is not to say that texture is not important in color perception and aesthetics. Texture does qualitatively change our perception of color. Texture characteristics, in part, lead to the distinction between surface and film color (Beck, 1975). Certainly, in the present study, the texture condition was the most satisfying to the subjects. Color appearance in that condition was phenomenally different from that of the other two; when the color was "right," the object seemed to "jump out" at the viewer. While latencies were not measured, texture matches also seemed to occur faster. Thus, texture seems to contribute to our perception of appearance, but as an attribute independent of color.

In contrast to the results reported by Bartleson and Bray (1962), we have shown no differences between memory colors and preferred colors. Bartleson and Bray attributed the differences they found to differences in context between two types of conditions. Memory colors were selected from an array of color patches, whereas color preferences were determined through judgments of photographs or objects varying in color. In the present study, one type of context, shape and texture information, was found not to be related to memory and preference differences. Context must be further differentiated in order to account for both sets of data. General context (a ring of fruits and vegetables surrounding the test area) was provided in this study in order to reduce what we have called the "house paint" effect. After choosing color from a brochure of color swatches, one can be quite surprised by the appearance of that color when it covers an entire house. It may well be that integrated context information (e.g., shape and texture) is not necessary for color-memory selection when a general context, providing relative size comparisons, is given. If general context providing accurate information is important for accurate color memory, one may wonder if providing a somewhat inaccurate color label may not be similar to providing an inaccurate context.

The speculations of early investigators concerning differences among observers based on their knowledge of color and color technology were not confirmed in the present study. While experts may be more critical observers, this does not seem to affect the nature or quality of their memory representations for the colors of objects. Experts do tend to show less variability in their color choices-a tendency that should be expected given their greater amounts of practice with tasks involving color selection and the use of a colorimeter.

Surprisingly, intrasubject variability was highly consistent across conditions. Within-subject variance differences seem to be related only to problems with specific fruits and vegetables, particularly the desaturated ones, and to procedural variations. Due to changes in uncertainty, one might have expected both memory and preference variances to be greater than matching variances and intrasubject variance differences due to changes in the contextual cues provided (disk, silhouette, or texture), but this was not the case. Neither a reduction in contextual cues nor the lack of a given standard in the memory and preference conditions produced an increase in intrasubject variance.

The data presented here indicate that, for some objects, memory for hue and brightness is quite accurate, and preferred hue and.brightness are those that are remembered. Only for saturation do remembered and preferred colors differ from actual color: objects are remembered and preferred as being more saturated than they actually are. These results were unaffected by the removal of shape and texture context information or by the expertness of the individuals making the color judgments. Such results are more consistent with a theory of memory representation in which the color of an object is stored as an independent attribute rather than one in which color is an integral part of a prototypic representation.

\section{REFERENCES}

Adnмs, G. K. An experimental study of memory color and related phenomena. American Journal of Psychology, 1923, 34, 359-407. 
BartLeson, C. J. Some observations on the reproduction of flesh colors. Photographic Science and Engineering, 1959, 3, 114-117.

BARTLEson, C. J. Memory colors of familiar objects. Journal of the Optical Society of America, 1960, 50, 73-77.

Bartleson, C. J. Color in memory in relation to photographic reproduction. Photographic Science and Engineering, 1961, 5, 327-331.

Bartleson, C. J., \& Bray, C. P. On the preferred reproduction of flesh, blue-sky, and green-grass colors. Photographic Science and Engineering, 1962, 6, 19-25.

BECK, J. The perception of surface color. Scientific American, $1975,233,62-75$.

Bornstein, M. H. Name codes and color memory. American Journal of Psychology, 1976, 89, 269-279.

Bruner, J. S., Postman, L., \& Rodrigues, J. Expectation and the perception of color. American Journal of Psychology, 1951, 64, 216-227.

Burnham, R. W. A colorimeter for research in color perception. American Journal of Psychology, 1952, 65, 603-608.

Duncker, K. The influence of past experience upon perceptual properties. American Journal of Psychology, 1939, 52, 255-265.

GARNER, W. G. The processing of information and structure. Potomac, Md: Erlbaum, 1974.

Hanes, R. M., \& Rhodes, M. V. Color identification as a function of extended practice. Journal of the Optical Society of America, 1959, 49, 1060-1064.

Harper, R. S. The perceptual modification of colored figures. American Journal of Psychology, 1953, 66, 86-89.

Hendrick, C., Wallace, G., \& TAPpenbeck, J. Effect of cognitive set on color perception. Journal of Personality and Social Psychology, 1968, 10, 487-494.

Hering, E. Outlines of a theory of the light sense (L. M. Hurvich \& D. Jameson, trans.). Cambridge, Mass: Harvard University Press, 1964.

JudD, D. B. A flattery index for artificial illuminants. Illuminating Engineering, 1967, 62, 593-598.

KATz, D. The world of colour (R. B. MacLeod \& C. W. Fox, trans). London: Kegan Paul, Trench, Trubner, 1935.

LofTus, E. F. Shifting human color memory. Memory \& Cognition, 1977, 5, 696-699.
MacAdam, D. L. Quality of color reproduction. Journal of the Society of Motion Picture and Television Engineers, 1951, 56, 487-512.

MYERS, J. L. Fundamentals of experimental design (3rd ed.). Boston: Allyn \& Bacon, 1979.

Newhall, S. M., Burnham, R. W., \& Clark, J. R. Comparison of successive with simultaneous color matching. Journal of the Optical Society of America, 1957, 47, 43-56.

SANDERs, C. L. Color preferences for natural objects. Illuminating Engineering, 1959, 54, 452-456.

Springer, R. M. Time errors in color memory. Unpublished master's thesis, California State University at Los Angeles, 1969.

Sugimoto, M., Ezawa, M., \& Tamura, T. A color converter and its application for measuring human color preferences in color television. Journal of the Society for Motion Picture and Television Engineers, 1973, 82, 71-77.

Tate, J. D., \& Springer, R. M. Effects of memory time on successive judgments. Psychological Bulletin, 1971, 76, 394-408.

Thonnton, W. A. A validation of the color-preference index. Illuminating Engineering, 1974, 4, 48-52.

\section{NOTES}

1. While we use Munsell terminology throughout the paper, the reader should substitute "brightness" and "saturation" for "value" and "chroma" if those terms hold more meaning.

2. The sampling distribution of variances, unlike the sampling distribution of means, is somewhat positively skewed. This type of nonnormality should have little effect on the ANOVAs carried out on variances in this paper, since ns are equal and there is no reason to believe that the shapes of the population distributions are different (Myers, 1979). Some authors recommend a logarithmic transformation for standard deviations and other positively skewed distributions, since the tendency of these distributions is to increase Type I error. In all of the variance analyses reported here, no meaningful, significant results are found, even with this possibly inflated probability of error.

(Manuscript received January 14, 1983; revision accepted for publication June 22,1983 .) 